\title{
Epidural hematoma occurred by massive bleeding intraoperatively in cesarean section after combined spinal epidural anesthesia -A case report-
}

\author{
Ji-Hyun Chung, Jinhwan Hwang, Seung-Cheol Cha, Taehyeng Jung, and Seong Chang Woo
}

Department of Anesthesiology and Pain Medicine, Eulji University School of Medicine, Daejeon, Korea

We describe a case of acute lumbar epidural hematoma at the L2-3 level complicated by paraplegia, which occurred after coagulation disorder because of massive bleeding intraoperatively in cesarean section. The preoperative coagulation laboratory finding was in normal range and so we tried combined spinal epidural anesthesia. Uterine atony occurred in the operation, and there was persistant bleeding during and after the operation. After the operation, she complained of paresthesia on her both legs and was diagnosed with epidural hematoma (EDH) by radiologic examination. Emergency laminectomy on lumbar spine was carried out for hematoma evacuation and decompression of the epidural space at once. In our experience, massive bleeding during surgery may potentially increase the risk of EDH postoperatively. (Korean J Anesthesiol 2011; 61: 336-340)

Key Words: Blood coagulation disorder, Cesarean section, Epidural anesthesia, Epidural hematoma, Postpartum hemorrhage, Spinal anesthesia.

Usually, patients are more satisfied following regional anesthesia of the lower abdomen or lower extremities than with general anesthesia; thus, regional anesthesia is often used during a cesarean section. Due to the advantages of rapid induction of spinal anesthesia and epidural anesthesia for pain control after surgery, the frequency of combined spinal-epidural anesthesia in pregnant women is also increasing. Damage to the epidural vein during epidural or spinal anesthesia occurs frequently, but the bleeding is usually temporary and mild, which stops immediately through a patient's normal coagulation system. Therefore, the incidence of EDH after epidural or spinal anesthesia is considered to be very rare [1]. In most cases, the use of anticoagulants before and after surgery was the major cause of EDH rather than mechanical injury of the vessels [2]. However, we experienced a case of acute EDH in a pregnant patient with a normal coagulation system who

Received: March 2, 2011. Revised: April 29, 2011. Accepted: May 3, 2011.

Corresponding author: Seong Chang Woo, M.D., Department of Anesthesiology and Pain Medicine, Eulji University School of Medicine, 1306, Dunsan 2-dong, Seo-gu, Daejeon 302-799, Korea. Tel: 82-42-611-3655, Fax: 82-42-611-3882, E-mail: woonoh@eulji.ac.kr

(c) This is an open-access article distributed under the terms of the Creative Commons Attribution Non-Commercial License (http:// creativecommons.org/licenses/by-nc/3.0/), which permits unrestricted non-commercial use, distribution, and reproduction in any medium, provided the original work is properly cited. 
had developed a coagulation disorder due to massive bleeding during the cesarean section. Herein, we report this case with a review of the pertinent literature.

\section{Case Report}

The patient was a 30-year-old primipara with a twin pregnancy at 36 weeks. She had an accompanying premature rupture of the membrane and had planned to have a cesarean section. She did not have a past medical history and her height and weight was $164 \mathrm{~cm}$ and $69 \mathrm{~kg}$, respectively. The physical examination, blood test, urine analysis, chest x-ray, and EKG were all within normal limits (Table 1). Combined spinalepidural anesthesia was planned because the patient wanted regional anesthesia for the twin pregnancy and there were with no special contraindications.

Preanesthesia medication was not used. EKG monitoring, non-invasive automatic blood pressure measuring, and pulse oximetry were set up after the patient arrived at the surgical theatre. The patient's blood pressure was 130/80 mmHg; heart rate was 90 beats per minute before induction. $3 \mathrm{~L}$ of oxygen was provided through nasal prong while observing the patient's vital signs. She was positioned left lateral decubitus for the regional anesthesia using an $18 \mathrm{G}$ modified Tuohy $\left(\right.$ Espocan $^{\circledR}$, B. Brown, Germany) needle, and epidural access between the third and fourth lumbar vertebrae was attempted. Right after the puncture, bleeding was observed through the needle, and the puncture needle was immediately removed. Compression was attempted for bleeding control. Since there was no observed bleeding or hematoma in the puncture area, a second attempt at puncture was tried between the second and third lumbar vertebrae with a $27 \mathrm{G}$ subarachnoid needle inserted in an $18 \mathrm{G}$ epidural needle after examining the epidural area with the loss of resistance technique. After observing spontaneous flow of CSF fluid, $5 \mathrm{mg}$ of $0.5 \%$ bupivacaine dextrose was injected, and then, the subarachnoid needle was removed. The subarachnoid needle bevel was toward the cephalic, with a $20 \mathrm{G}$ epidural catheter (Perifix $^{\circledR}$ soft tip, Braun, Germany) located $3 \mathrm{~cm}$ from the cephalic. Then, the patient was repositioned to the supine position. There were no difficulties during the procedure, and she did not complain of any dysesthesia, numbness, or pain. After no blood or CSF had been aspirated, a $3 \mathrm{ml}$ test amount of epinephrine $(1: 200,000)$ was injected through the epidural catheter with a 3 -minute observation period. The patient was stable with blood pressure at $130 / 80 \mathrm{mmHg}$ and heart rate at 95 beats per minute. 10 minutes after intraspinal injection, the level of the sensory block was at the tenth thoracic spinal nerve segment. In order to increase the level of the sensory block, after checking again that no blood had been aspirated, $5 \mathrm{ml}$ of $2 \%$ lidocaine mixed with epinephrine $(1: 200,000)$ was injected repeatedly with a total of $20 \mathrm{ml}$ being injected. After 20 minutes, the level of the sensory block did not change remaining at the tenth thoracic spinal nerve segment. Therefore, it became necessary to switch to general anesthesia. $250 \mathrm{mg}$ of Thiopental and $75 \mathrm{mg}$ of succinylcholine were infused. After observed loss of consciousness and muscle relaxation, intubation was done. While the surgery was proceeding, the anesthesia was maintained using $2 \mathrm{~L} / \mathrm{min}$ of $\mathrm{O}_{2}, 2 \mathrm{~L} / \mathrm{min}$ of $\mathrm{N}_{2} \mathrm{O}, 5$ vol\% of desflurane, and $4 \mathrm{mg}$ of vecuronium until the babies were delivered. 4 minutes after induction, the baby boy was delivered through cesarean section, and the baby girl was delivered 1 minute after the birth of the boy. The babies weighed $2.4 \mathrm{~kg}$ and $2.6 \mathrm{~kg}$ with 5-minute-apgar scores of 7 and 8 . After the placenta was expelled, 20 units of oxytocin mixed with $100 \mathrm{ml}$ of normal saline were infused, but uterine contraction did not successfully proceed. Bleeding of more than 3,000 $\mathrm{ml}$ occurred in the 5 minutes after delivery. $0.2 \mathrm{mg}$ of methylergonovine was injected to advance uterine contraction, but the bleeding continued. Her hemoglobin level dropped to $8.4 \mathrm{mg} / \mathrm{dl}$. Since massive bleeding was not expected before operation, there was no blood prepared. One hour after the operation, 2 units of packed RBCs prepared under emergency conditions were transfused. Crystalloid solution and colloid solution was infused before the transfusion with repeated injections of ephedrine to maintain systolic blood pressure above $90 \mathrm{mmHg}$. After the operation, blood pressure was maintained above 90/40 $\mathrm{mmHg}$ without an inotropic or vasopressor agent. Total anesthetic time was 2 hours 10 minutes; the operation time was 1 hour 20 minutes.

Table 1. Laboratory Finding

\begin{tabular}{|c|c|c|c|c|c|c|}
\hline & \multirow{2}{*}{ Pre-operation } & \multirow{2}{*}{ Intra-operation } & \multicolumn{4}{|c|}{ Post-operation } \\
\hline & & & $1 \mathrm{~h}$ & $4 \mathrm{~h}$ & $12 \mathrm{~h}$ & $2 \mathrm{~d}$ \\
\hline Hemoglobin concentration (mg/dl) & 10.5 & 8.6 & 10.1 & 7.1 & 8.6 & 9.6 \\
\hline Hematocrit (\%) & 33.3 & 24 & 29.1 & 20.4 & 24.4 & 27.2 \\
\hline Platelet count $\left(\times 10^{3} / \mathrm{ml}\right)$ & 166 & & 117 & 82 & 79 & 118 \\
\hline activated partial thromboplastin time (s) & 37.8 & & 52.3 & 46.4 & 46.4 & 50.9 \\
\hline prothrombin time $(\mathrm{s})$ & 13.9 & & 21.1 & 19.3 & 19.3 & 17 \\
\hline activity (\%) & 90 & & 43 & 50 & 50 & 61 \\
\hline International normalized ratio & 1.07 & & 1.84 & 1.64 & 1.64 & 1.39 \\
\hline
\end{tabular}


Total bleeding during anesthesia was approximately 4,000 ml; urine amount was approximately $200 \mathrm{ml}$, and 2 units of packed RBCs were transfused. 1,000 ml crystalloid and 2,000 ml colloid solutions were infused. In the recovery room, the patient received an extra 2 units of packed RBCs and 2 units of fresh frozen plasma were transfused.

After arriving at the recovery room, the level of the sensory block was below T12. She was transferred to the ward after 1 hour after partial recovery of sensory and motor function was observed and the epidural catheter was removed. Right after arriving at the ward, a blood test was done; hemoglobin was $10.1 \mathrm{mg} / \mathrm{dl}$ with prolonged PT and aPTT each measured at 21.1 sec and $52.3 \mathrm{sec}$, respectively. Vaginal bleeding continued after arriving at the ward. Intrauterine compression was attempted by foley catheter insertion and 2 units of fresh frozen plasma were transfused. 4 hours after the operation, PT and aPTT were $19.3 \mathrm{sec}$ and $46.4 \mathrm{sec}$. Hemoglobin level decreased to $7.1 \mathrm{mg} / \mathrm{dl}$ and platelet count decreased to $82,000 / \mathrm{mm}^{3} .3$ units of packed RBCs and 10 units of platelet concentrate were transfused. The vaginal bleeding continued but decreased.

At the ward, the patient complained of abdominal pain and back pain but was able to get some sleep by controlling pain through patient-controlled-analgesia (PCA). Next morning, 10 hours after the operation, the back pain continued, and sensory and motor paralysis on both lower extremities were observed. Emergency magnetic resonance imaging (MRI) was done and a compressive mass was found at the posterior of the lumbar vertebrae (Fig. 1). Acute epidural hematoma was diagnosis, and an infusion of high dose steroid therapy was carried out.

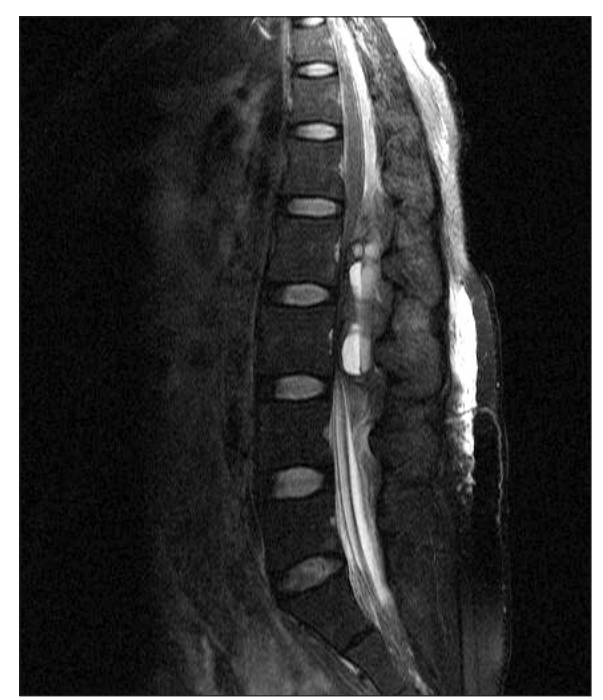

Fig. 1. Initial T2 weighted image demonstrate a high signal intense epidural hematoma with fluid-fluid level that extends from L2 to L3 and is posterior to the spinal cord, producting severe compression of distal spinal cord and proximal thecal sac.
The patient was transferred to the surgical theatre 4 hours after symptoms developed. An emergency decompression laminectomy was done to get rid of the epidural hematoma at the L2/L3 segment of the lumbar vertebrae. The patient's coagulation process did not work well and more than 1,000 $\mathrm{ml}$ of bleeding occurred with 3 units of packed RBCs being transfused. After the operation, motor and sensory function of the lower extremities did not change but the pain in the back had mildly improved. However, 1 day after the hematoma was removed, the back pain had become aggravated. Another emergency MRI was done and the hematoma was found to have occurred again, and the operation was done again. She complained of excessive numbness in her lower extremities but muscle strength gradually recovered. One month after the operation, she was discharged after she was able to walk without any working aid with some restrictions. Five months after the operation, neurological symptoms were totally improved without any sequelae.

\section{Discussion}

The advantages of regional anesthesia during cesarean section are reducing pulmonary aspiration of stomach contents, having no difficulties in maintaining airways due to intubation failure, and avoiding fetal suppression caused by general anesthesia. However, regional anesthesia can also cause various complications. It is rare but severe sequelae can occur depending on the patients. EDH after spinal anesthesia or epidural anesthesia can cause severe complications like paraplegia and urinary incontinence, for which immediate management is required since it can lead to permanent sequela.

Needle puncture or catheter insertion through vertebra can cause damage to the epidural vein leading to bleeding. However, most people with a normal coagulation system usually have minimal bleeding and hemostasis occurs immediately. Therefore, the frequency of epidural hematoma after spinal or epidural anesthesia does not usually occur. According to obstetric research, 1 out of 183,000 patients developed EDH, which is extremely rare [3]. In addition, pregnancy is defined as a mild hypercoagulation state, which reduces the risk of EDH. In practice, it was found that pregnant women have a lower risk of developing spontaneous EDH than normal adults [2]. $\mathrm{EDH}$ is related more to hemorrhagic disease with the use of anticoagulation or it is more like hemophilia [4].

As mentioned above, although EDH is an extremely rare complication, our patient had $\mathrm{EDH}$, and we were able postulate three possible causes of EDH by reviewing other research.

The first cause could be from the massive bleeding during delivery. It is usually expected to have $500 \mathrm{ml}$ of bleeding during vaginal delivery and 1,000 $\mathrm{ml}$ of bleeding during cesarean 
section. However, at the terminal stage of pregnancy, cardiac output increases by $50 \%$ and stroke volume increases by $25 \%$ with a total of $70-100 \mathrm{ml} / \mathrm{kg}$ of blood gain occurring. These modifications of the cardiovascular system during pregnancy provide maternity protection from bleeding complications. However, life-threatening conditions due to massive bleeding happen frequently. Most common causes of massive bleeding within 24 hours after delivery are uterine atony, retained placenta, injury of genital track, and inversion of uterus. If bleeding does not stop after the appropriate management, emergency hysterectomy is considered [5]. The frequency of hysterectomy after cesarean section is definitely higher than in vaginal delivery ( 2.36 out 1,000 cases versus 0.2 out 1,000 cases, respectively) [6]. Massive bleeding during delivery is a dangerous event, which can cause coagulation disorders like disseminated intravascular coagulation. The Patient in our case had massive bleeding during surgery, and the consumption of coagulation factors had occurred, which may have led to coagulation disorder resulting in $\mathrm{EDH}$.

The second cause could be the venous structures, which become vulnerable to mechanical damage due to dilation of the venous branch between the epidural space of the vertebrae and vertebrae body during pregnancy [7]. The frequency of vessel damage due to the catheter during epidural anesthesia is $3-9 \%$ [8]. The reason for this is that pregnancy is one of the risk factors that has a higher frequency in developing EDH. Although it is known that the hypercoagulation state during pregnancy suppresses the formation of a hematoma, the risk of vessel damage is higher. Spontaneous EDH in pregnant women has been continuously reported $[9,10]$.

The third cause could be from epidural needle puncture and from insertion and removal of the catheter into the epidural space of the vertebrae. According to Vandermeulen et al. [11], not only needle puncture and catheter insertion but also removal of the catheter also directly injures vessels and obviously influence the occurrence of bleeding. According to this report, more than half of the hematomas occur within 24 hours after the catheter has been removed. Other authors have also mentioned that the timing of the catheter removal, that is, the catheter is removed at a time when the patient's coagulation mechanism may not be normal, is a major risk factor of $\mathrm{EDH}[11,12]$. In the present case, the catheter was removed after the surgery in the recovery room. At that time, the patient was regionally anesthetized and sensory and motor functions could not be checked at that time. Neither the timing of the induction of the epidural and spinal anesthesia nor the timing of the catheter removal can be eliminated as causes of activate bleeding.

As mentioned above, epidural vessel damage commonly occurs, but it rarely causes problems when patients have a normal coagulation system. Instead, there is a reported case of
EDH 9 days after stimulation of the epidural space [7]. Which means re-bleeding after trauma is possible without mechanical stimulation due to coagulopathy. In the present case, pregnancy caused an anatomical condition that was vulnerable to mechanical damage; stimulation of the epidural space by insertion and removal of the catheter during anesthesia induction are causes of vascular injury, and massive bleeding during the operation induced by coagulopathy caused rebleeding and made bleeding control difficult.

EDH commonly causes ventral lumbar pain and radiating pain and induces functional impairment of the sensory and motor nerve; it also causes spine compression, cauda equina syndrome combined with quadriplegia or paraplegia, and myelopathy combined with bladder dilation; therefore, computed tomography (CT), myelography, and MRI should be done for immediate and appropriate treatment. With CT, it is hard to differentiate EDH from other diseases that compress dura mater; myelography can differentiate tumor shape, size, and location, but it has to use invasive contrast medium. However, MRI is an non-invasive procedure, which can differentiate epidural and subarachnoid hemorrhage, herniated disc, and spinal cord edema in spine injured patients with neurological disorders; it is the best way to exam hematomas and to exam the severity of nerve compression confirming the damage around the surrounding soft tissue [13].

In order to minimize permanent nerve damage, early surgical decompression of the hematoma is recommended as a treatment modality for EDH. It is best to have the operation before paralysis or within a few hours after paralysis has occurred to prevent irreversible ischemia [1,2]. There are some reports of spontaneous recovery from $\mathrm{EDH}[14,15]$; however, considering the pathological characteristics of a hematoma being clotted, surgical removal seems to be the most reasonable course to follow. Many reports have stated it is best to have surgery within $24-36$ hours after symptoms manifest. In the present case, the patient was able to fully recover due to immediate management.

Delayed recovery from regional anesthesia and use of analgesia like PCA for extreme abdominal pain after delivery may cause confusion because it can postpone a patient's complaints of symptoms. EDH can occur in patients without coagulopathy, mechanical stimulation, or trauma [13]. In order to prevent coagulopathy and to minimize complications, close attention should be given to the complaints of patients who had epidural or spinal anesthesia for delivery during recovery.

\section{References}

1. Dickman CA, Shedd SA, Spetzler RF, Shetter AG, Sonntag VK. Spinal epidural hematoma associated with epidural anesthesia: 
Complications of systemic heparinization in patients receiving peripheral vascular thrombolytic therapy. Anesthesiology 1990; 72: 947-50.

2. Kreppel D, Antoniadis G, Seeling W. Spinal hematoma: a literature survey with meta-analysis of 613 patients. Neurosurg Rev 2003; 26 : 1-49.

3. Ruppen W, Derry S, McQuay H, Moore RA. Incidence of epidural hematoma, infection, and neurologic injury in obstetric patients with epidural analgesia/anesthesia. Anesthesiology 2006; 105: 3949.

4. Vandermeulen EP, Vermylen J, Aken HV. Epidural and spinal anaesthesia in patients receiving anticoagulant therapy. Baillieres Clin Anaesthesiol 1993; 7: 663-89.

5. Banks A, Norris A. Massive haemorrhage in pregnancy. Contin Educ Anaesth Crit Care Pain 2005; 5: 195-8.

6. Ahn HK, Kim ES, Han JY, Kim MY, Ryu HM, Choi KH, et al. Emergency peripartum hysterectomy: a five year review. Korean J Obstet Gynecol 2001; 44: 2190-3.

7. Guffey PJ, Mckay WR, Mckay RE. Epidural hematoma nine days after removal of a labor epidural catheter. Anesth Analg 2010; 111: 992-5.
8. Verniquet AJ. Vessel puncture with epidural catheters: Experience in obstetric patients. Anaesthesia 1980; 35: 660-2.

9. Bose S, Ali Z, Rath GP, Prabhakar H. Spontaneous spinal epidural haematoma: a rare cause of quadriplegia in the post-partum period. Br J Anaesth 2007; 99: 855-7.

10. Szkup P, Soneham G. Case report: spontaneous spinal epidural haematoma during pregnancy: case report and review of the literature. Br J Radiol 2004; 77: 881-4.

11. Vandermeulen EP, Van Aken H, Vermylen J. Anticoagulants and spinal-epidural anesthesia. Anesth Analg 1994; 79: 1165-77.

12. Miyazaki M, Takasita M, Matsumoto $H$, Sonoda $H$, Tsumura $H$, Torisu T. Spinal epidural hematoma after removal of an epidural catheter: case report and review of the literature. J Spinal Disord Tech 2005; 18: 547-51.

13. Lee JS, Suh KT. Spontaneous lumbar spinal epidural hematoma without risk factors. J Korean Orthop Assoc 2005; 40: 1013-6.

14. Tailor J, Dunn IF, Smith E. Conservative treatment of spontaneous spinal epidural hematoma associated with oral anticoagulant therapy in a child. Childs Nerv Syst 2006; 22: 1643-5.

15. Silber SH. Complete nonsurgical resolution of a spontaneous spinal epidural hematoma. Am J Emerg Med 1996; 14: 391-3. 